\title{
Clinical features of severe Mycoplasma pneumoniae pneumonia in adults admitted to an intensive care unit
}

\author{
Correspondence \\ Naoyuki Miyashita \\ nao@med.kawasaki-m.ac.jp
}

Received 12 December 2006

Accepted 30 July 2007

\author{
Naoyuki Miyashita, ${ }^{1}$ Yasushi Obase, ${ }^{1}$ Kazunobu Ouchi, ${ }^{2}$ Kozo Kawasaki, ${ }^{2}$ \\ Yasuhiro Kawai, ${ }^{2}$ Yoshihiro Kobashi ${ }^{1}$ and Mikio Oka ${ }^{1}$ \\ ${ }^{1}$ Division of Respiratory Diseases, Department of Medicine, Kawasaki Medical School, Okayama \\ 701-0192, Japan \\ ${ }^{2}$ Department of Pediatrics, Kawasaki Medical School, Kurashiki, Okayama 701-0192, Japan
}

Community-acquired pneumonia (CAP) due to Mycoplasma pneumoniae is usually mild, but some cases develop a severe life-threatening pneumonia. To investigate the clinical features of severe $M$. pneumoniae pneumonia in adults admitted to an intensive care unit, a multi-centre CAP surveillance study was performed. Among all hospitalized CAP cases between January 2000 and December 2004, there were 227 cases with M. pneumoniae pneumonia without the complication of other pathogens. A total of 13 of the cases required admission to an intensive care unit because of acute respiratory failure (ARF), and the remaining 214 cases (non-ARF) were low to moderately severe. The clinical features of ARF cases were compared with those of non-ARF cases. The underlying conditions in both types of case were identical, whereas clinical findings on admission clearly differed between the two groups. A regimen of an antibiotic effective against $M$. pneumoniae was begun on average at 9.3 days after the onset of symptoms in ARF cases, which was significantly later than for non-ARF cases $(P<0.0001)$. However, two of the ARF cases progressed to respiratory failure despite the fact that adequate antibiotics were initially administered within 3 days after the onset of symptoms. All ARF cases received corticosteroids with adequate antibiotics, and their condition improved promptly. These results indicate that the clinical features, excluding underlying conditions, clearly differed between severe M. pneumoniae pneumonia and low to moderately severe pneumonia. The delayed administration of adequate antibiotics may contribute to the severity of $M$. pneumoniae pneumonia. Early corticosteroid therapy with adequate antibiotics should be considered.

\section{INTRODUCTION}

Community-acquired pneumonia (CAP) is common and sometimes becomes fatal. Despite substantial progress in therapeutic options, CAP remains a significant cause of morbidity and mortality worldwide. Mycoplasma pneumoniae is one of the most common causes of CAP, accounting for as many as $10-30 \%$ of all cases of CAP (Blasi, 2004; Miyashita et al., 2005; Waites \& Talkington, 2004). In Japan, M. pneumoniae has been the third leading pathogen causing CAP, but it is becoming the second. NIHIDSC (2007) demonstrated that the incidence of $M$. pneumoniae pneumonia in recent years is about 10 -fold higher than before 1999 (on average 0.45 cases per selected hospital weekly in 2006 and $<0.05$ cases per selected hospital

Abbreviations: ALT, alanine aminotransferase; ARF, acute respiratory failure; AST, aspartate aminotransferase; CAP, community-acquired pneumonia; $\mathrm{FiO}_{2}$, fraction of inspired $\mathrm{O}_{2} ; \mathrm{IL}$, interleukin; $\mathrm{LDH}$, lactate dehydrogenase; $\mathrm{PaO}_{2}$, partial pressure of $\mathrm{O}_{2}$ in arterial blood; Th1, Thelper type 1 ; TP, total protein. weekly before 1999) (NIHIDSC, 2007). Since pneumonia due to M. pneumoniae is usually mild, the term 'walking pneumonia' has been widely used by physicians (Waites \& Talkington, 2004). However, some studies have shown $M$. pneumoniae to be second only to Streptococcus pneumoniae in hospitalized adults (Marston et al., 1997; Miyashita et al., 2002; Porath et al., 1997), and 3-4\% of those infected with M. pneumoniae are known to develop a severe lifethreatening pneumonia with respiratory failure and acute respiratory distress syndrome (Chan \& Welsh, 1995; Fraley et al., 1979; Koletsky \& Weinstein, 1980). Of patients who were admitted to the hospital, up to $10.9 \%$ required mechanical ventilation (Marrie, 1993; Waites \& Talkington, 2004). In Japan, only some cases of fulminant pneumonia with acute respiratory failure (ARF) and fatal cases caused by $M$. pneumoniae infection have been reported (Ito et al., 1995; Takiguchi et al., 2001; Tsuruta et al., 2002). Since epidemiological studies have demonstrated that the incidence of $M$. pneumoniae pneumonia is gradually increasing (NIHIDSC, 2007), severe M. pneumoniae 
pneumonia may not be uncommon, but is probably underdiagnosed owing to a lack of awareness or an underestimation of such cases. Furthermore, severe pneumonia due to $M$. pneumoniae has not been investigated in Japan. In addition, there are no comparative studies that have investigated the clinical features of low to moderately severe pneumonia versus severe pneumonia due to $M$. pneumoniae. The purpose of this study was to clarify the clinical features of severe $M$. pneumoniae pneumonia in adult patients admitted to an intensive care unit. We performed a multi-centre CAP surveillance study and investigated M. pneumoniae pneumonia in adults.

\section{METHODS}

Study population. All adult patients with CAP who were admitted to Okayama Kyoritsu Hospital, Kurashiki Daiichi Hospital, Kurashiki Central Hospital, Mizushima Kyodo Hospital, Kawasaki Medical School Kawasaki Hospital and Kawasaki Medical School Hospital, Okayama, Japan, from January 2000 to December 2004, were enrolled in this study. None of the patients were immunocompromised, that is, they were not patients with HIV infection, with neutropenia secondary to chemotherapy nor on immunosuppressants, the patients were not from nursing homes, nor had they been recently $(<30$ days) admitted to hospital. The diagnosis was based on clinical signs and symptoms (cough, fever, productive sputum, dyspnoea, chest pain or abnormal breath sounds), and radiographic pulmonary abnormalities that were at least segmental and were due to pre-existing or other known causes. All cases of pneumonia occurring more than 3 days after hospitalization were considered nosocomial and were excluded. Informed consent was obtained from all subjects; the study protocol was approved by the Ethics Committee at Kawasaki Medical School.

Microbiological laboratory tests. Blood cultures and nasopharyngeal swab specimens were obtained from all patients on admission and, if pleural fluid and sputum were available, a Gram stain test and a quantitative culture were obtained. Sputum data were only evaluated when the Gram stain test revealed numerous leukocytes $(>25$ in one $\times 100$ microscope field) and few squamous epithelial cells ( $<10$ in one $\times 100$ microscope field). Certain invasive methods, such as bronchoscopic examination, were employed to obtain specimens from all patients that underwent endotracheal intubation and some non-intubated patients after full explanation of the procedures. These specimens were also used for culturing of $M$. pneumoniae and Legionella species on pleuropneumonia-like organism broth and agar [70\% pleuropneumonia-like organism broth (Difco) supplemented with $20 \%$ heat-inactivated horse serum, $10 \%$ fresh yeast extract $(25 \%)$, thallium acetate (final concentration $0.5 \mathrm{mg} \mathrm{ml}^{-1}$ ) and sterile penicillin $\mathrm{G}$ (final concentration $1000 \mathrm{U}$ $\mathrm{ml}^{-1}$ )] and buffered charcoal-yeast extract alpha agar, respectively. Cultures for Chlamydophila pneumoniae and Chlamydophila psittaci were performed in cycloheximide-treated HEp-2 cells grown in a 24well cell culture plate (Miyashita et al., 2005). All specimens were passed twice. Culture confirmation was done by fluorescent-antibody staining with Chlamydophila pneumoniae and Chlamydophila psittaci species-specific and genus-specific mAbs (Miyashita et al., 2005). The bronchoscopic specimens were also used for PCR of M. pneumoniae and Chlamydia species. The M. pneumoniae-specific primers used for the PCR were from the DNA base sequence within the P1 cytadhesin gene and amplification was performed as reported by Ramirez et al. (1996). The Chlamydophila pneumoniae-specific and genus-specific primers used for PCR were from the DNA base sequence within the $53 \mathrm{kDa}$ protein gene and major outer-membrane protein gene, respectively, established in our laboratory (Miyashita et al., 2002).
This assay was performed as previously described (Miyashita et al., 2002).

Paired serum samples were collected at intervals of at least 4 weeks after onset. Complement fixation tests were done in all patients for antibodies to influenza $\mathrm{A}$ and $\mathrm{B}$ viruses, adenovirus, respiratory syncytial virus, cytomegalovirus, and parainfluenza virus types 1,2 and 3. Antibodies to M. pneumoniae were measured by the passive agglutination test (Serodia-Myco II kit; Fujirebio), Legionella species by the microagglutination test (detection of Legionella pneumophila serogroups 1-6, Legionella bozemanae, Legionella dumoffii, Legionella gormanii and Legionella micdadei) and Coxiella burnetii by the indirect immunofluorescence test. The microimmunofluorescence test was used for titration of IgG and IgM antibodies against chlamydial species, using formalinized elementary bodies of Chlamydophila pneumoniae KKpn-15, Chlamydia trachomatis L2/ 434/Bu and Chlamydophila psittaci Budgerigar-1 strains as antigens (Miyashita et al., 2002). Rheumatoid factors were absorbed with Gullsorb (Gull Laboratories) before IgM titrations. In addition to serology, culturing and/or PCR, the urinary antigen test (BinaxNOW; Binax) was used for detection of S. pneumoniae and L. pneumophila.

Criteria for determination of microbial aetiology. The microbial aetiology was classified as 'definitive', 'presumptive' or 'unknown'. Bacteria were considered to be definitive causative agents when isolated from blood or pleural fluid cultures. We considered the results of sputum cultures in combination with Gram stain findings. An organism showing heavy $\left(\geqslant 10^{7}\right.$ c.f.u. $\left.\mathrm{ml}^{-1}\right)$ or moderate $\left(10^{6}\right.$ c.f.u. $\mathrm{ml}^{-1}$ ) growth of a predominant bacterium on a sputum culture was considered to be a presumptive pathogen. Any micro-organism isolated from bronchoscopic specimens was considered to be a presumptive pathogen when its concentration reached $>10^{5}$ c.f.u. $\mathrm{ml}^{-1}$ in quantitative culture. If $M$. pneumoniae or the Legionella species were isolated from a specimen, that specimen was considered to be a definitive pathogen even if the culture showed little growth. $L$. pneumophila and S. pneumoniae were considered to be a presumptive agent when the urinary antigen test was positive. For serological tests, a fourfold rise in the antibody titre level between paired sera was considered definitive. Acute Chlamydophila pneumoniae infection was defined as IgM $\geqslant 1: 32$ or a fourfold rise in IgG or IgM titre between acute and convalescent serum samples.

M. pneumoniae pneumonia cases. The patients who fulfilled the diagnostic criteria for pneumonia caused by M. pneumoniae without any evidence of other causative agents were divided into two groups, of cases with ARF and those without ARF, for comparison of the clinical features. Among all CAP cases, there were 227 cases in which $M$. pneumoniae was the only pathogen identified by the panel of diagnostic tests used. Cases of $M$. pneumoniae pneumonia mixed with other micro-organisms were excluded in this study. Thirteen cases who showed $\mathrm{PaO}_{2} / \mathrm{FiO}_{2}<200$ and bilateral diffuse infiltrates on chest radiographs during hospitalization required admission to an intensive care unit (ARF cases). Of these, nine cases underwent endotracheal intubation, and mechanical ventilation was established. The remaining 214 cases had low to moderately severe pneumonia without ARF (non-ARF cases), which was judged by the severity score according to Japanese CAP guidelines (Committee for the JRS Guidelines in Management of Respiratory Infections, 2005). The Pneumonia Severity Index risk classification showed them to be in classes II to IV (Fine et al., 1997). All M. pneumoniae cases demonstrated fourfold antibody seroconversion (42 cases were culture positive). Among the 13 ARF cases, culture and PCR using bronchoscopic specimens were performed in all cases and 6 cases, respectively. Eight cases were culture positive and six cases were PCR positive.

Statistical analysis. Statistical analysis for the incidence of underlying conditions and clinical findings was done by Fisher's 
exact test. A mean age and laboratory data comparison was done by the Student's $t$ test.

\section{RESULTS AND DISCUSSION}

Table 1 shows the underlying conditions and clinical findings on admission to hospital. Chan \& Welsh (1995) reviewed 46 cases of fulminant $M$. pneumoniae pneumonia that resulted in respiratory failure or death, and concluded that fulminant cases seem to be more common in young healthy adults, in males, and, possibly, in smokers. In our comparative study, ARF cases were also observed in young healthy adults (mean age 38 years), but no significant differences in age were observed between ARF cases and non-ARF cases. Gender differences were not observed in the two groups and the frequency of a smoking history was also identical in both groups. Thus, younger age, no comorbidities, gender and a smoking history do not seem to be true risks for increasing the severity of M. pneumoniae pneumonia.

In contrast to underlying conditions, laboratory findings, including the mean white blood cell count and the mean serum levels of C-reactive protein, total protein (TP), lactate dehydrogenase (LDH), alanine aminotransferase (ALT) and aspartate aminotransferase (AST) on admission clearly differed in the ARF and non-ARF cases. Chest radiography findings also differed. Six ARF cases showed bilateral diffuse infiltrates on chest radiographs on admission. Unilateral infiltrates appeared on chest radiographs of the remaining seven ARF cases on admission, but progressed to bilateral diffuse infiltrates after admission.
Fraley et al. (1979) noted that bilateral pulmonary infiltrates, pleural effusion, leukocytosis and extrapulmonary complications seem to be characteristic of severe $M$. pneumoniae infection. Our results indicated that in addition to these, elevated levels of LDH, ALT and AST, and a reduced level of TP, seem to be characteristic of severe M. pneumoniae pneumonia.

Table 2 shows the time (mean no. of days) required for several clinical conditions after the onset of clinical symptoms due to $M$. pneumoniae infection, and Table 3 shows the treatment regimen against $M$. pneumoniae pneumonia before and after hospitalization. An important difference was observed between the ARF cases and nonARF cases with regard to the administration of adequate antibiotics active against $M$. pneumoniae. A regimen of an antibiotic effective against $M$. pneumoniae was begun on average at 9.3 days after the onset of symptoms in ARF cases but it was begun significantly later than that in nonARF cases $(P<0.0001)$. Chan \& Welsh $(1995)$ reported that the effective regimen of an antibiotic against M. pneumoniae was begun on average at 10 days after onset of symptoms in cases with nonfatal respiratory failure and at 15 days after in cases with fatal respiratory failure. Thus, delay in the administration of adequate antibiotics is one of the most important reasons for the development of ARF.

However, two of our ARF cases progressed to respiratory failure in spite of the initial administration of adequate antibiotics within 3 days after the onset of symptoms. In contrast, seven of the non-ARF cases received adequate antibiotics at $>10$ days after the onset of symptoms, but none of them progressed to respiratory failure.

Table 1. Underlying conditions and clinical findings on hospital admission of 227 patients with M. pneumoniae pneumonia

Data represent numbers (SD).

\begin{tabular}{|c|c|c|c|}
\hline Characteristic & ARF $(n=13)$ & Non-ARF $(n=214)$ & $P$ value \\
\hline Male: female & $7: 6$ & $109: 105$ & $>0.9999$ \\
\hline Smoking history & 4 & 70 & $>0.9999$ \\
\hline Comorbidities & 4 & 34 & 0.2401 \\
\hline Respiratory rate $\left(>30 \mathrm{~min}^{-1}\right)$ & 11 & 0 & $<0.0001$ \\
\hline Pulse rate $\left(>125 \mathrm{~min}^{-1}\right)$ & 4 & 9 & 0.0035 \\
\hline Systolic blood pressure $(<90 \mathrm{mmHg})$ & 3 & 0 & 0.0001 \\
\hline $\mathrm{PaO}_{2}<7998 \mathrm{~Pa}$ & 13 & 17 & $<0.0001$ \\
\hline Mean no. of white blood cells $\left(\times 1000 \mu \mathrm{l}^{-1}\right)$ & $16.2(9.5)$ & $7.2(2.1)$ & $<0.0001$ \\
\hline Mean level C-reactive protein $\left(\mathrm{mg} \mathrm{dl}^{-1}\right)$ & $27.3(8.4)$ & $10.1(7.9)$ & $<0.0001$ \\
\hline Mean level TP $\left(\mathrm{mg} \mathrm{dl}^{-1}\right)$ & $5.6(0.6)$ & $7.1(0.5)$ & $<0.0001$ \\
\hline Mean level LDH $\left(\mathrm{IU} \mathrm{l}^{-1}\right)$ & $750(428)$ & $254(111)$ & $<0.0001$ \\
\hline Mean level ALT (IU 1 ${ }^{-1}$ ) & $64.8(52.2)$ & $30.0(26.1)$ & $<0.0001$ \\
\hline Mean level AST $\left(\mathrm{IU} \mathrm{l}^{-1}\right)$ & $67.2(38.7)$ & $29.5(17.3)$ & $<0.0001$ \\
\hline \multicolumn{4}{|l|}{ Chest radiograph findings ${ }^{*}$} \\
\hline Unilateral infiltrates & 0 & 171 & $<0.0001$ \\
\hline Bilateral infiltrates & 13 & 43 & $<0.0001$ \\
\hline Pleural effusion & 5 & 5 & $<0.0001$ \\
\hline
\end{tabular}

${ }^{\star}$ Chest radiograph findings during hospitalization. 
Table 2. The time required for several clinical conditions after onset of clinical symptoms due to M. pneumoniae infection

Data represent the mean number of days after onset of symptoms (SD).

\begin{tabular}{|lrrr|}
\hline Clinical condition & ARF $(\boldsymbol{n}=\mathbf{1 3})$ & $\begin{array}{r}\text { Non-ARF } \\
(\boldsymbol{n}=\mathbf{2 1 4})\end{array}$ & $\boldsymbol{P}$ value \\
\hline Initial antibiotic administration before admission to hospital $^{*}$ & $4.5(3.0)$ & $3.2(2.6)$ & 0.0971 \\
Adequate antibiotic administration & $9.3(3.2)$ & $5.1(2.9)$ & $<0.0001$ \\
Admission to hospital & $6.5(3.2)$ & $5.3(2.6)$ & 0.1475 \\
Endotracheal intubation & $10.6(3.6)$ & & \\
Steroid administration & $10.0(3.4)$ & & \\
\hline
\end{tabular}

${ }^{*}$ Including either adequate or inadequate antibiotics.

Unfortunately, no differences were found in any clinical conditions between ARF cases and non-ARF cases who received adequate antibiotics at $>10$ days after the onset of symptoms. Thus, deterioration of the patients was not only due to delayed administration of adequate antibiotics.

It has been shown that the cell-mediated immunity of the host plays an important role in the development of $M$. pneumoniae pneumonia (Chan \& Welsh, 1995; Evengard et al., 1994; Foy et al., 1973; Waites \& Talkington, 2004). In a mouse model, Tanaka et al. (1996) reported that interleukin (IL)-2 administration [stimulating T-helper type (Th) 1 reaction] exacerbated peribronchial and perivascular cuffing of lymphocytes and plasma cells, and led to enlargement of nodular lesions consisting of macrophage accumulation within bronchioles. Cyclosporin A administration, which inhibits the transcription

Table 3. Regimen of treatment against $M$. pneumoniae pneumonia before and after hospitalization

Data represent numbers of cases.

\begin{tabular}{|lcr|}
\hline Characteristic & $\begin{array}{c}\text { ARF } \\
(\boldsymbol{n}=\mathbf{1 3})\end{array}$ & $\begin{array}{r}\text { Non-ARF } \\
(\boldsymbol{n}=\mathbf{2 1 4})\end{array}$ \\
\hline Initial treatment before hospitalization & & \\
$\beta$-lactams & 6 & 95 \\
Macrolides & & 15 \\
Fluoroquinolones & & 9 \\
No antibiotic & 7 & 95 \\
After hospitalization & 9 & 10 \\
$\beta$-lactams & & 201 \\
Minocycline & 2 & 2 \\
Minocycline + carbapenems & 2 & 1 \\
Macrolides + carbapenems & & \\
After endotracheal intubation & 2 & \\
Minocycline + carbapenems & 1 & \\
Minocycline + fluoroquinolones & 5 & \\
Macrolides + carbapenems & 1 & \\
Macrolides + fluoroquinolones & & \\
\hline
\end{tabular}

of IL-2 and gamma interferon, however, reduced these pulmonary lesions. Using mice with different immunogenetic backgrounds (BALB/c mice vs $\mathrm{C} 57 \mathrm{BL} / 6$ mice), Fonseca-Aten et al. (2005) found that M. pneumoniae induces host-dependent pulmonary and immunological responses. In addition, Tanaka et al. (2002) demonstrated that the levels of serum IL-18, which promotes Th1 cytokine responses, in patients with severe $M$. pneumoniae pneumonia were higher than those in mild cases, and that they, but not serum gamma interferon levels, correlated with the number of affected lobes detected by computed tomography scans. Kitching et al. (2000) reported that, even in the absence of IL-12, IL-18 could induce an in vivo delayed-type hypersensitivity response. The delayed-type hypersensitivity response to mycoplasma organisms appears to correlate with the severity of pneumonia (Mizutani et al., 1971). These results suggest that an excessive host-cellular response with Th1 cytokines and IL18 may play a critical role in the development of worse pulmonary manifestations.

Corticosteroids downregulate the cell-mediated immune response and therefore may have a profound effect by reducing the immune-mediated pulmonary injury seen in mycoplasmal infections. Clinically, corticosteroids have been used for severe M. pneumoniae pneumonia, and indeed advocated for decreasing the inflammatory response and improving survival (Chan \& Welsh, 1995; Fraley et al., 1979; Ito et al., 1995; Koletsky \& Weinstein, 1980; Takiguchi et al., 2001; Tsuruta et al., 2002). All our ARF cases received high doses of corticosteroids in addition to adequate antibiotics and their condition improved promptly. Nine out of thirteen ARF cases received corticosteroids after intubation. Early administration of corticosteroids might have prevented mechanical ventilation. These findings support the early use of corticosteroids in addition to adequate antibiotic therapy for severe $M$. pneumoniae infections.. Although administration of steroids may be associated with an increased risk of new infections and adverse effects on metabolism, their use seems to be justified for patients who are developing respiratory failure. 
In conclusion, our results indicate that a younger age, no comorbidities, gender and a smoking history do not seem to be true risks for increasing the severity of $M$. pneumoniae pneumonia. Leukocytosis, bilateral pulmonary infiltrates, pleural effusion, elevated levels of LDH, ALT and AST, and a reduced level of TP, seem to be characteristic in severe $M$. pneumoniae pneumonia. Current evidence from human and animal studies suggests that individual immune responses to M. pneumoniae infection may vary, and that the severity of the clinical illness and pulmonary injury may correlate directly with a more vigorous cell-mediated immune response and cytokine stimulation. In addition to cellmediated immunity, a delayed administration of adequate antibiotics ( $>10$ days after onset of symptoms) may contribute to the severity of $M$. pneumoniae pneumonia.

\section{ACKNOWLEDGEMENTS}

We gratefully acknowledge members of the Kurashiki Chest Conference Group (Okayama Kyoritsu Hospital, Kurashiki Daiichi Hospital, Kurashiki Central Hospital, Mizushima Kyodo Hospital and Kawasaki Medical School Kawasaki Hospital) for providing details of M. pneumoniae pneumonia. This work was supported by the MEXT KAKENHI (19591190), 32nd Kanae Foundation for Life and Socio-Medical Science, and project research grants from Kawasaki Medical School (13-401, 14402, 15-405A, 16-405M, 17-402M, 18-401, 19-402M).

\section{REFERENCES}

Blasi, F. (2004). Atypical pathogens and respiratory tract infections. Eur Respir J 24, 171-181.

Chan, E. D. \& Welsh, C. H. (1995). Fulminant Mycoplasma pneumoniae pneumonia. West J Med 162, 133-142.

Committee for the JRS Guidelines in Management of Respiratory Infections (2005). The JRS Guidelines for the Management of Community-Acquired Pneumonia in Adults (in Japanese). Edited by T. Matsushima, S. Kohno \& A. Saito. Tokyo: Japanese Respiratory Society.

Evengard, B., Sandstedt, K., Bolske, G., Feinstein, R., RiesenfeltOm, I. \& Smith, C. I. (1994). Intranasal inoculation of Mycoplasma pulmonis in mice with severe combined immunodeficiency (SCID) causes a wasting disease with grave arthritis. Clin Exp Immunol 98, 388-394.

Fine, M. J., Auble, T. E., Yealy, D. M., Hanusa, B. H., Weissfeld, L. A., Singer, D. E., Coley, C. M., Marrie, T. J. \& Kapoor, W. N. (1997). A prediction rule to identify low-risk patients with community-acquired pneumonia. $N$ Engl J Med 336, 243-250.

Fonseca-Aten, M., Rios, A. M., Mejias, A., Chavez-Bueno, S., Katz, K., Gomez, A. M., McCracken, G. H., Jr \& Hardy, R. D. (2005), Mycoplasma pneumoniae induces host-dependent pulmonary inflammation and airway obstruction in mice. Am J Respir Cell Mol Biol 32, 201-210.

Foy, H. M., Ochs, H., Davis, S. D., Kenny, G. E. \& Luce, R. R. (1973). Mycoplasma pneumoniae infection in patients with immunodeficiency syndromes: report of four cases. J Infect Dis 127, 388-393.

Fraley, D. S., Ruben, F. L. \& Donnelly, E. J. (1979). Respiratory failure secondary to Mycoplasma pneumoniae infection. South Med J 72, $437-440$
Ito, S., Abe, Y., Kinomoto, K., Saitoh, T., Kato, T., Kohli, Y., Kuriyama, M., Sakai, T. \& Ishizaki, T. (1995). Fulminant Mycoplasma pneumoniae pneumonia with marked elevation of serum soluble interleukin-2 receptor. Intern Med 34, 430-435.

Kitching, A. R., Tipping, P. G., Kurimoto, M. \& Holdsworth, S. R. (2000). IL-18 has IL-12 independent effects in delayed-type hypersensitivity: studies in cell-mediated crescentic glomerulonephritis. J Immunol 165, 4649-4657.

Koletsky, R. J. \& Weinstein, A. J. (1980). Fulminant Mycoplasma pneumoniae infection. Report of a fatal case, and review of the literature. Am Rev Respir Dis 122, 491-496.

Marrie, T. J. (1993). Mycoplasma pneumoniae pneumonia requiring hospitalization, with emphasis on infection in the elderly. Arch Intern Med 153, 488-494.

Marston, B. J., Plouffe, J. F., File, T. M., Jr, Hackman, B. A., Salstrom, S.-J., Lipman, H. B., Kolczak, M. S. \& Breiman, R. F. (1997). Incidence of community-acquired pneumonia requiring hospitalization. Results of a population-based active surveillance study in Ohio. The Community-Based Pneumonia Incidence Study Group. Arch Intern Med 157, 1709-1718.

Miyashita, N., Fukano, H., Okimoto, N., Hara, H., Yoshida, K., Niki, Y. \& Matsushima, T. (2002). Clinical presentation of communityacquired Chlamydia pneumoniae pneumonia in adults. Chest 121, 1776-1781.

Miyashita, N., Fukano, H., Mouri, K., Fukuda, M., Yoshida, K., Kobashi, Y., Niki, Y. \& Oka, M. (2005). Community-acquired pneumonia in Japan: a prospective ambulatory and hospitalized patient study. J Med Microbiol 54, 395-400.

Mizutani, H., Kitayama, T., Hayakawa, A. \& Nagayama, E. (1971). Delayed hypersensitivity in Mycoplasma pneumoniae infection. Lancet 1, 186-187.

NIHIDSC (2007). Mycoplasma pneumoniae pneumonia. National Institute of Health Infectious Disease Surveillance Center, Tokyo, Japan. http://idsc.nih.go.jp/idwr/kanja/weeklygraph/18myco-e.html.

Porath, A., Schlaeffer, F. \& Lieberman, D. (1997). The epidemiology of community-acquired pneumonia among hospitalized adults. J Infect 34, 41-48.

Ramirez, J. A., Ahkee, S., Tolentino, A., Miller, R. D. \& Summersgill, J. T. (1996). Diagnosis of Legionella pneumophila, Mycoplasma pneumoniae, or Chlamydia pneumoniae lower respiratory infection using the polymerase chain reaction on a single throat swab specimen. Diagn Microbiol Infect Dis 24, 7-14.

Takiguchi, Y., Shikama, N., Aotsuka, N., Koseki, H., Terano, T. \& Hirai, A. (2001). Fulminant Mycoplasma pneumoniae pneumonia. Intern Med 40, 345-348.

Tanaka, H., Honma, S., Abe, S. \& Tamura, H. (1996). Effects of interleukin-2 and cyclosporine A on pathologic features in Mycoplasma pneumonia. Am J Respir Crit Care Med 154, 1908-1912.

Tanaka, H., Narita, M., Teramoto, S., Saikai, T., Oashi, K., Igarashi, T. \& Abe, S. (2002). Role of interleukin-18 and T-helper type 1 cytokines in the development of Mycoplasma pneumoniae pneumonia in adults. Chest 121, 1493-1497.

Tsuruta, R., Kawamura, Y., Inoue, T., Kasaoka, S., Sadamitsu, D. \& Maekawa, T. (2002). Corticosteroid therapy for hemolytic anemia and respiratory failure due to Mycoplasma pneumoniae pneumonia. Intern Med 41, 229-232.

Waites, K. B. \& Talkington, D. F. (2004). Mycoplasma pneumoniae and its role as a human pathogen. Clin Microbiol Rev 17, 697-728. 